\title{
The Impact of the Pulmonary Artery Index and Aortopulmonary Collateral Artery Coil Embolization on Intractable Pleural Effusions After a Fontan Surgery
}

\author{
Taisuke Nabeshima, ${ }^{1,2}$ MD, Yuichi Ishikawa, ${ }^{1}$ MD, Naokata Sumitomo, ${ }^{2}$ MD, Kiyotaka Go, ${ }^{3}$ MD, \\ Yoshihiko Kodama, ${ }^{1}$ MD, Ayako Kuraoka, ${ }^{1}$ MD, Makoto Nakamura, ${ }^{1}$ MD, \\ Koichi Sagawa, ${ }^{1} \mathrm{MD}$ and Toshihide Nakano, ${ }^{4} \mathrm{MD}$
}

\begin{abstract}
Summary
An intractable pleural effusion is a common comorbidity of a Fontan operation, occasionally leading to undesirable outcomes. The preventive effect of aortopulmonary collateral (APC) coil embolization against a pleural effusion before a Fontan operation is still controversial.

This is a retrospective single-center study; among 227 Fontan cases, 57 cases with complete MRI data were analyzed at first. Factors associated with the duration of pleural drainage (median: 6 (2-41) days) and that of postoperative hospital stay (median: 25 (14-91) days) were analyzed using a multiple regression analysis. The pulmonary artery index (PAI; Nakata index) was associated with both the pleural drainage duration $\left(P<0.05, r^{2}\right.$ $=0.17)$ and postoperative hospital stay $\left(P<0.05, r^{2}=0.10\right)$.

Thereafter, all the 227 patients were classified into the following three groups: Group A (12 patients in whom the embolization was performed within 30 days before the Fontan surgery), Group B (131 patients in whom the embolization was performed more than 30 days before the Fontan surgery), and Group C (84 patients in whom the embolization was not performed). Patients in Group A were found to be associated with the shortest length of both periods $(P<0.05)$.

Lower PAI values were related to a prolonged pleural drainage duration and postoperative hospital stay. APC coil embolizations may reduce the risk if they are performed shortly (less than 30 days) before the operation.
\end{abstract}

(Int Heart J 2021; 62: 559-565)

Key words: Fontan procedure, Pleural drainage, Postoperative hospital stay, Adaptation to Fontan circulation, Early outcomes

$\mathrm{T}$ he Fontan operation was first performed in 1971, and the surgical techniques and perioperative management have progressed over the past four decades. As a result, the early- and long-term outcomes of the Fontan operation have dramatically improved. In our institute, over the last 20 years before 2014, the early and late mortality rates were $0.4 \%$ and $3.4 \%$, respectively. ${ }^{1)}$

However, some complications may progress early after a Fontan operation, such as chylothorax and prolonged pleural effusions, which may lead to poor nutrition and vulnerability to infections and result in a longer hospital stay and higher in-hospital mortality rates. ${ }^{2)}$ Various factors related to persistent pleural effusions following a Fontan operation were reported, such as a lower preoperative oxygen saturation, the presence of postoperative infections or surgery during the respiratory viral season, a smaller conduit size, and a longer duration of the cardiopulmon- ary bypass. ${ }^{3-6)}$ Although it has been said that the preFontan aortopulmonary collateral (APC) flow correlates with persistent pleural drainage and lengths of longer hospital stays, ${ }^{7)}$ the impacts of the APC coil embolization prior to a Fontan operation on pleural effusions or length of hospital stay remain obscure.

The aim of this study was to clarify the risk factors of intractable pleural effusions after a Fontan operation and whether an APC coil embolization before surgery could possibly contribute to lowering the risk of a pleural effusion.

\section{Methods}

This study was approved by the institutional review board of Fukuoka Children's Hospital (2019-68), and written informed consent was waived because of the retro-

From the ${ }^{1}$ Department of Pediatric Cardiology, Fukuoka Children's Hospital, Fukuoka, Japan, ${ }^{2}$ Department of Pediatric Cardiology, Saitama Medical University, International Medical Center, Saitama, Japan, ${ }^{3}$ Department of Peditric Cardiology, Nagoya University Graduate School of Medicine, Aichi, Japan and ${ }^{4}$ Department of Pediatric Cardiovascular Surgery, Fukuoka Children's Hospital, Fukuoka, Japan.

Address for correspondence: Taisuke Nabeshima, MD, Department of Pediatric Cardiology, Saitama Medical University, International Medical Center, 1397-

1, Yamane, Hidaka, Saitama, 350-1298, Japan. E-mail: taisuke_nabeshima@icloud.com

Received for publication July 16, 2020. Revised and accepted December 15, 2020.

Released in advance online on J-STAGE May 15, 2021.

doi: 10.1536/ihj.20-498

All rights reserved by the International Heart Journal Association. 


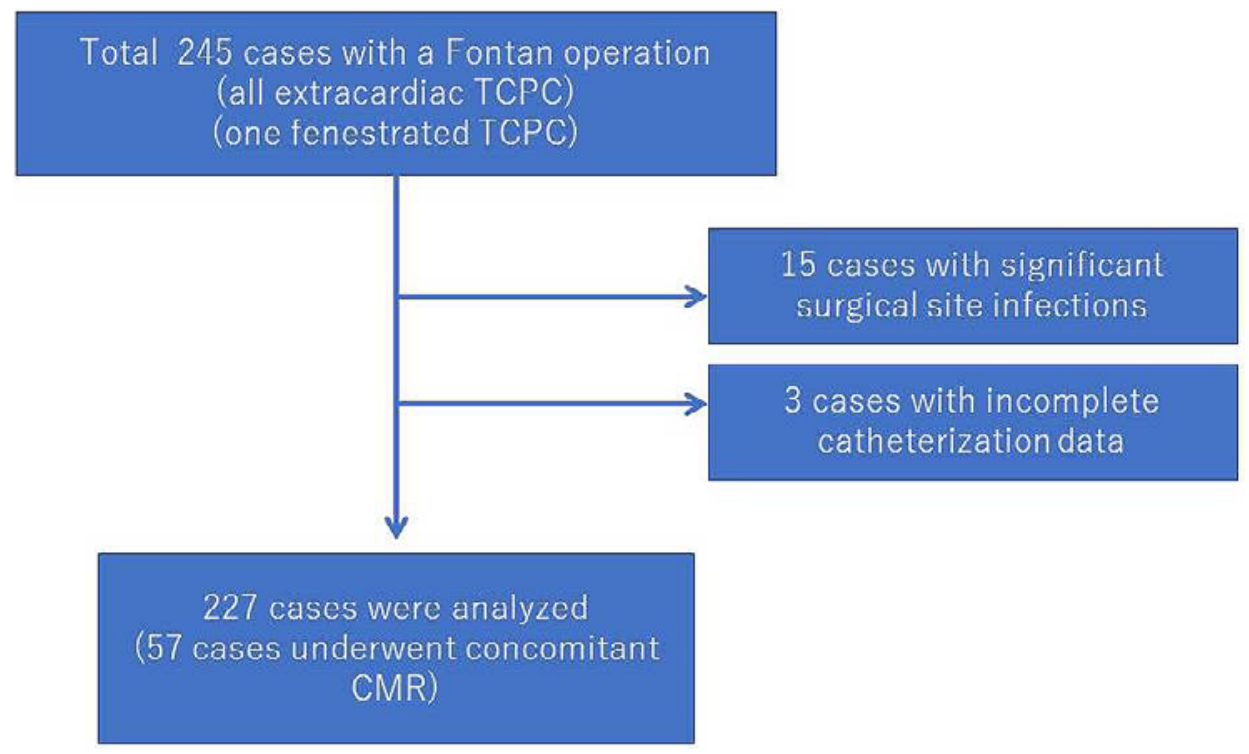

Figure 1. Summary of the included and excluded cases (see the text for the details).

spective design.

Study population: Two hundred forty-five consecutive patients who underwent a Fontan operation at our institute between January 2009 and December 2017 were retrospectively assessed. Fifteen patients who were subjected to prolonged antibiotic use (> 14 days) or surgical debridement due to surgical-site infections were excluded, and another 3 patients with incomplete pre-Fontan catheterization data were also excluded. Among the remaining 227 patients, all patients underwent cardiac catheterization before the Fontan operation, and 57 patients additionally underwent cardiac magnetic resonance imaging (MRI) (Figure 1). The patent demographics are detailed in Table I.

Measurement: The primary outcomes or dependent variables were the duration of the pleural drainage and postoperative hospital stay length. Although we did not have any definite criteria for the removal of the pleural drainage, we typically removed it when the amount of effusion remained below $2 \mathrm{~mL} / \mathrm{kg} /$ day for 7 days with medications and there was little concern of any further pleural effusion accumulation. If we needed further drainage during the same hospitalization, the total number of days with drainage was also counted.

Some independent variables were extracted from the parameters thought to represent the hemodynamics of bidirectional Glenn circulation such as the partial pressure of the arterial oxygen $\left(\mathrm{SaO}_{2}\right)$, mean pulmonary artery pressure (mPAP), pulmonary artery index (PAI; Nakata index: defined as the sum of the cross-sectional area of left and right pulmonary arteries $\left(\mathrm{mm}^{2}\right)$ divided by the body surface area $\left.\left(\mathrm{m}^{2}\right)\right){ }^{8)}$ systemic ventricular ejection fraction (SVEF), the ratio of the pulmonary flow and systemic flow (Qp/Qs), \% systemic-pulmonary collateral flow (SPCF), and pulmonary resistance (Rp) index. Among those, $\mathrm{SaO}_{2}, \mathrm{mPAP}, \mathrm{PAI}$, and SVEF were obtained by means of cardiac catheterization, whereas $\mathrm{Qp} / \mathrm{Qs}$ and \% SPCF were obtained by cardiac MRI. The Rp index was calculated using both catheterization and cardiac MRI data as follows: $\mathrm{Rp}$ index $=(\mathrm{mPAP}($ catheter $)-$ LA pressure (catheter)) / Qp(cardiac MRI). The details of each value are presented in Table I.

Protocol of cardiac MRI: We typically performed cardiac MRI and cardiac catheterizations around 6 months before the Fontan operations. The flow volumes of each vessel (i.e., superior vena cava $[\mathrm{SVC}]$, inferior vena cava $[\mathrm{IVC}]$, aorta [Ao], pulmonary arteries [PAs], and pulmonary veins $[\mathrm{PVs}]$ ) were obtained using two-dimensional phase-contrast MRI (1.5-T Avanto Magneton; Siemens Medical, Erlangen, Germany).

The SPCF was measured using two methods; the SPCF1 was defined as $\mathrm{Q}_{\mathrm{Ao}}-\left(\mathrm{Q}_{\mathrm{svc}}+\mathrm{Q}_{\mathrm{Ivc}}\right)$, and the SPCF 2 as $\mathrm{Q}_{\mathrm{PV}}-\left(\mathrm{Q}_{\mathrm{LPA}}+\mathrm{Q}_{\mathrm{RPA}}\right)$. The \% $\mathrm{SPCF}$ was defined as SPCF/Qp.

Cardiac catheterization and cardiac MRI were performed under room air conditions. However, we routinely provided home oxygen therapy soon after the bidirectional Glenn operation based on the clinical implication that oxygen might have favorable effects on the bidirectional Glenn circulation by suppressing the sympathetic tone and lowering pulmonary vascular resistance.9)

Protocol of the APC coil embolization: We evaluated the amount of APC based on an aortogram ${ }^{10)}$ and instantly discussed the necessity for coil embolization in each case. Although there are no definite criteria for performing coil embolization, we typically perform it in cases where APCs are so significant that there are apparent overloads to the main ventricles. The effect of the embolization was evaluated using angiography at the end of each procedure. The end-point of the embolization was determined using the amount of the APC decreased at the end of the embolization. However, the timing of the embolization was attributed to the interventionist and circumstances of each patient.

Investigation of the effectiveness of APC coil embolization: All patients were classified into three groups accord- 
Table I. Patient Characteristics (Overall)

\begin{tabular}{|c|c|c|}
\hline & Median & First-Third IQRs \\
\hline Age at Fontan operation (years) & 3.18 & $2.48-3.76$ \\
\hline Body weight at Fontan operation (kg) & 11.8 & $11.1-12.7$ \\
\hline Gender (Male/Female) (cases) & $136 / 91$ & \\
\hline \multicolumn{3}{|l|}{ Diagnostic Category } \\
\hline Diagnosis (HLHS) (cases) & 49 & \\
\hline Diagnosis (SRV) (cases) & 77 & \\
\hline Diagnosis (SLV) (cases) & 84 & \\
\hline Diagnosis (BV) (cases) & 17 & \\
\hline \multicolumn{3}{|l|}{ Medication } \\
\hline Pulmonary Vasodilator (cases) & 19 & \\
\hline ACE inhibitor (cases) & 202 & \\
\hline Beta blocker (cases) & 61 & \\
\hline \multicolumn{3}{|l|}{ Pre-Fontan hemodynamic data } \\
\hline $\mathrm{SaO} 2(\%)$ & 87 & $85-88$ \\
\hline mPAP (mmHg) & 8 & $7-10$ \\
\hline PAI $\left(\mathrm{mm}^{2} / \mathrm{m}^{2}\right)$ & 245 & $187-302$ \\
\hline $\mathrm{EF}(\%)$ & 58 & $53-63$ \\
\hline $\mathrm{CI}\left(\mathrm{L} / \mathrm{minute} / \mathrm{m}^{2}\right)$ & 3.2 & $2.8-3.7$ \\
\hline Qp/Qs & 1.02 & $0.85-1.15$ \\
\hline$\%$ SPCF1 (\%) & 42.2 & $30.2-47.6$ \\
\hline$\% \operatorname{SPCF} 2(\%)$ & 40.4 & $30.0-49.8$ \\
\hline RpI (Wood) & 1.37 & $1.10-1.75$ \\
\hline \multicolumn{3}{|l|}{ Pre-Fontan Echocardiography } \\
\hline AVVR grade $\geq 2$ (cases) & 32 & \\
\hline AVVR grade < 2 (cases) & 195 & \\
\hline \multicolumn{3}{|l|}{ Pre-Fontan APC embolization } \\
\hline Performed < 30 days (cases) & 12 & \\
\hline Performed > 30 days (cases) & 131 & \\
\hline Not performed (cases) & 84 & \\
\hline \multicolumn{3}{|c|}{$\begin{array}{l}\text { The variables are listed as the actual number for the number of cases and as the median } \\
\text { followed by the 1st-3rd quartiles for the other variables. HLHS indicates hypoplastic lef } \\
\text { heart syndrome; SRV, single right ventricle (except HLHS); SLV, single left ventricle } \\
\text { BV, biventricle; mPAP, mean pulmonary artery pressure; PAI, pulmonary artery index } \\
\text { (Nakata index); SPCF, systemic-pulmonary collateral flow; RpI, pulmonary resistance } \\
\text { index; APC, aortopulmonary collateral vessels; and AVVR, atrioventricular valve regur- } \\
\text { gitation. }\end{array}$} \\
\hline
\end{tabular}

ing to the period between the APC coil embolization and Fontan surgery as follows: Group A included patients in whom an embolization was performed within 30 days before the surgery; Group B included patients in whom the embolization was performed 30 days or more before the surgery; and Group C included patients in whom no embolization was performed before the surgery. Differences between the groups were analyzed in terms of the duration of the pleural drain placement and length of the hospital stay after the surgery.

Statistical analysis: Continuous data are expressed as the median and interquartile range (IQR), first to third IQR. A multiple regression analysis was used to investigate factors associated with the duration of pleural drainage and length of the postoperative hospital stay. Those factors with significant correlation with others are dismissed, and the following variables are selected as independent variables: gender, body weight at Fontan operation, PAI, mPAP, EF, Qp/Qs, SPCF2, RpI, and Cp. Regression equations are deduced by a step-wise method. A one-way analysis of variance was used to compare the outcomes of the three groups classified by the duration between the APC embolization and Fontan surgery. A $P$-value $<0.05$ was con- sidered to be statistically significant. All statistical analyses were completed using commercial software (SPSS Statistics ${ }^{\circledR}$ version 23; IBM Corp., Armonk, NY, USA).

\section{Results}

Risk factors for intractable pleural effusions following a Fontan operation: The patient demographics and preFontan hemodynamics are summarized in Table I. The age and body weight at the time of the Fontan operation were 3.3 years and $12.2 \mathrm{~kg}$, respectively. As for the medication usage after the bidirectional Glenn operation, angiotensinconverting enzyme inhibitors, or angiotensin II receptor blockers together with home oxygen therapy were prescribed in nearly all cases. Conversely, we did not routinely employ pulmonary vasodilation. The median time between the catheterization and Fontan operation was 5 months.

Regarding the grade of atrioventricular valve regurgitation (AVVR) before the Fontan operation, 32 cases had more than a moderate grade (grade 2), and 195 case had less than a mild grade (grade 1). The median duration of the pleural drainage and length of the postoperative hospi- 
Table II. Results of the Multiple Regression Analysis

\begin{tabular}{|c|c|c|c|}
\hline & \multicolumn{2}{|c|}{ Unstandardized Coefficients } & \multirow{2}{*}{$\begin{array}{c}\text { Standardized } \\
\text { Coefficients } \\
\text { Beta }\end{array}$} \\
\hline & B & Std. Error & \\
\hline \multicolumn{4}{|c|}{ Y: Duration of pleural drainage placement $\mathrm{X}^{*}$} \\
\hline (Constant) & 17.0 & 3.1 & \\
\hline PAI & -0.03 & 0.012 & -0.33 \\
\hline \multicolumn{4}{|c|}{ Y: Length of postoperative hospital stay $\mathrm{X}^{* *}$} \\
\hline (Constant) & 55.8 & 6.7 & \\
\hline PAI & -0.09 & 0.025 & -0.43 \\
\hline
\end{tabular}

*The dependent variant $(\mathrm{Y})$ is the duration of pleural drainage placement, and the independent variable $(\mathrm{X})$ is PAI. $\mathrm{Y}=17.0-(0.03 \times \mathrm{PAI})\left(P=0.01, r^{2}=0.10\right)$. ** The dependent variant $(\mathrm{Y})$ is the length of postoperative hospital stay, and the independent variable $(\mathrm{X})$ is PAI. $\mathrm{Y}=55.8-(0.09 \times$ PAI) $\left(P=0.001, r^{2}=0.17\right) . r^{2}$ indicates the determination coefficient or contribution ratio.

tal stay were respectively 6 (4-10.5) and 26.5 (20.5-35.5) days in those with more than a grade 2 and 6 (4-9) and 25 (21-37) days in those with less than a grade 1 . There was no statistical difference between these two groups in terms of both outcomes ( $P=0.14$ and 0.07 , respectively).

The results of multiple regression analysis are presented in Table II. Regression equations are deduced as follows: the pleural drainage duration (days) $=17.0-$ $(0.03 \times \mathrm{PAI})\left(P=0.01, r^{2}=0.10\right)$, and postoperative hospital stay $($ days $)=55.8-(0.09 \times \mathrm{PAI})\left(P=0.001, r^{2}=\right.$ $0.17)$. Thus, it can be concluded that PAI is a sole factor associating with both the duration of pleural drainage and the length of postoperative hospital stay, although the contribution ratio $\left(r^{2}\right)$ is small.

Influence of the APC coil embolization before the Fontan operation: Although there was no recommendation regarding the use of the embolization, we did not perform embolization for APC circulation routinely in all preFontan patients. In total, 143 of 227 (63\%) patients underwent APC coil embolization, including 112 patients in whom it was conducted simultaneously with a hemodynamic assessment, whereas in the other 31 patients, it was performed independently.

To elucidate the influence of APC coil embolization before the Fontan operation, we divided the patients into three groups as follows: Group A (12 patitents in whom the embolization was performed within 30 days before the Fontan surgery), Group B (131 patients in whom the embolization was performed more than 30 days before the Fontan surgery), and Group C (84 patients in whom the embolization was not performed before the Fontan surgery). The median age and body weight at the time of Fontan surgery in each group are 2.98, 3.15, and 3.26 years $(P>0.05)$ and $11.4,11.8$, and $11.9 \mathrm{~kg}(P>0.05)$, respectively. Other baseline patient characteristics of each group are presented in Table III. There were no differences in the mPAP, PAI, and other background characteristics between these groups.

Figures 2, 3 illustrate differences between the groups with regard to the pleural drainage and postoperative length of hospital stay. The patients belonging to Group A were found to be associated with the shortest length of the pleural drainage and postoperative length of stay. All of the Fontan operations were conducted by means of extracardiac TCPC, with the exception of one patient with a fenestration.

There was one early-term death due to a massive hemothorax (belonging to Group B) and two late-term deaths (belonging to Group C). In the latter group, one patient passed away 5 months later after the surgery because of a low cardiac output syndrome and multiple organ failure, and another one presented with heterotaxy syndrome (asplenia) and Streptococcus pneumoniae sepsis more than 1 year later.

At 6 months of follow-up from the catheterization after the Fontan operation, most patients continued to exhibit relatively low CVP (mean: $10 \mathrm{mmHg}$ ) and high $\mathrm{SpO}_{2}$ (mean: $95 \%$ ) values.

\section{Discussion}

In our series, patients with smaller PAI before the Fontan surgery were at a higher risk of a post-Fontan intractable pleural effusion. As far as we know, this is the first study to have investigated the risk factors among the pre-Fontan hemodynamic parameters. Moreover, we found that APC coil embolization was effective as far as it was performed shortly before the Fontan surgery (less than 30 days).

Regarding the grade of AVVR and other factors, there is a report ${ }^{11}$ demonstrating that AVVR of more than grade 2 is a significant predictor of supraventricular tachycardia early after a Fontan operation, but we could not find any significant difference in terms of the pleural drainage placement and postoperative hospital stay. Besides, there is a possibility that avoiding positive pressure ventilation to achieve a lower intra-thoracic pressure soon after the surgery is beneficial. In our practice, we try to extubate as soon as possible after Fontan operations, which is a feasible option, unless there is a risk of airway trouble. ${ }^{12)}$

Clinical implications of the influence of PAI on persistent pleural effusions: After Fontan operations, IVC flow is added to the bidirectional Glenn pulmonary circulation, and pulmonary blood flow (Qp) increases significantly. We speculate that if the pulmonary vascular bed (represented by PAI and pulmonary vascular compliance (Cp)) is not abundant enough to accommodate the added flow, then the "overflowed" Qp would emerge as a persistent pleural effusion. In other words, according to the Starling 
Table III. Patient Demographics (Comparison of Three Groups)

\begin{tabular}{|c|c|c|c|}
\hline Demographics & $\begin{array}{c}\text { Group A } \\
\text { APC } \\
\text { embolization }(+) \\
<30 \text { days before } \\
\text { Fontan }\end{array}$ & $\begin{array}{c}\text { Group B } \\
\text { APC } \\
\text { embolization }(+) \\
>30 \text { days before } \\
\text { Fontan }\end{array}$ & $\begin{array}{c}\text { Group C } \\
\text { APC } \\
\text { embolization (-) } \\
\text { before Fontan }\end{array}$ \\
\hline Patient Number & 12 & 131 & 84 \\
\hline Gender (Male/Female) & $7 / 5$ & $79 / 52$ & $50 / 34$ \\
\hline \multicolumn{4}{|l|}{ Diagnostic Category } \\
\hline HLHS & $3(25 \%)$ & $34(26 \%)$ & $12(15 \%)$ \\
\hline SRV (except HLHS) & $4(33 \%)$ & $40(30 \%)$ & $33(39 \%)$ \\
\hline SLV & $4(33 \%)$ & $50(38 \%)$ & $30(23 \%)$ \\
\hline BV & $1(8 \%)$ & $7(5 \%)$ & $9(11 \%)$ \\
\hline Emboli-Fontan (days) & $29(4-25)$ & $166(101-236)$ & - \\
\hline $\mathrm{SaO}_{2}(\%)$ & $88(88-90)$ & $87(85-88)$ & $84(86-89)$ \\
\hline PA pressure $(\mathrm{mmHg})$ & $9(9-16)$ & $9(6-16)$ & $8(5-12)$ \\
\hline PA index $\left(\mathrm{mm}^{2} / \mathrm{m}^{2}\right)$ & $227(159-282)$ & $233(190-293)$ & $253(184-315)$ \\
\hline Age (years) at the Fontan & $2.98(2.37-3.43)$ & $3.15(2.44-3.69)$ & $3.26(2.56-3.90)$ \\
\hline Body weight $(\mathrm{kg})$ at the Fontan & $11.4(11.1-11.9)$ & $11.8(11.1-12.6)$ & $11.9(11.2-12.7)$ \\
\hline
\end{tabular}

Patients are classified into three groups. Group A: APC embolization performed within 30 days before the Fontan. Group B: APC embolization performed more than 30 days before the Fontan. Group C: without an APC embolization before the Fon$\tan$. There was no significant difference in the background characteristics between groups $(P>0.05)$. HLHS indicates hypoplastic left heart syndrome; SRV, single right ventricle (except HLHS); SLV, single left ventricle; and BV, Biventricle.

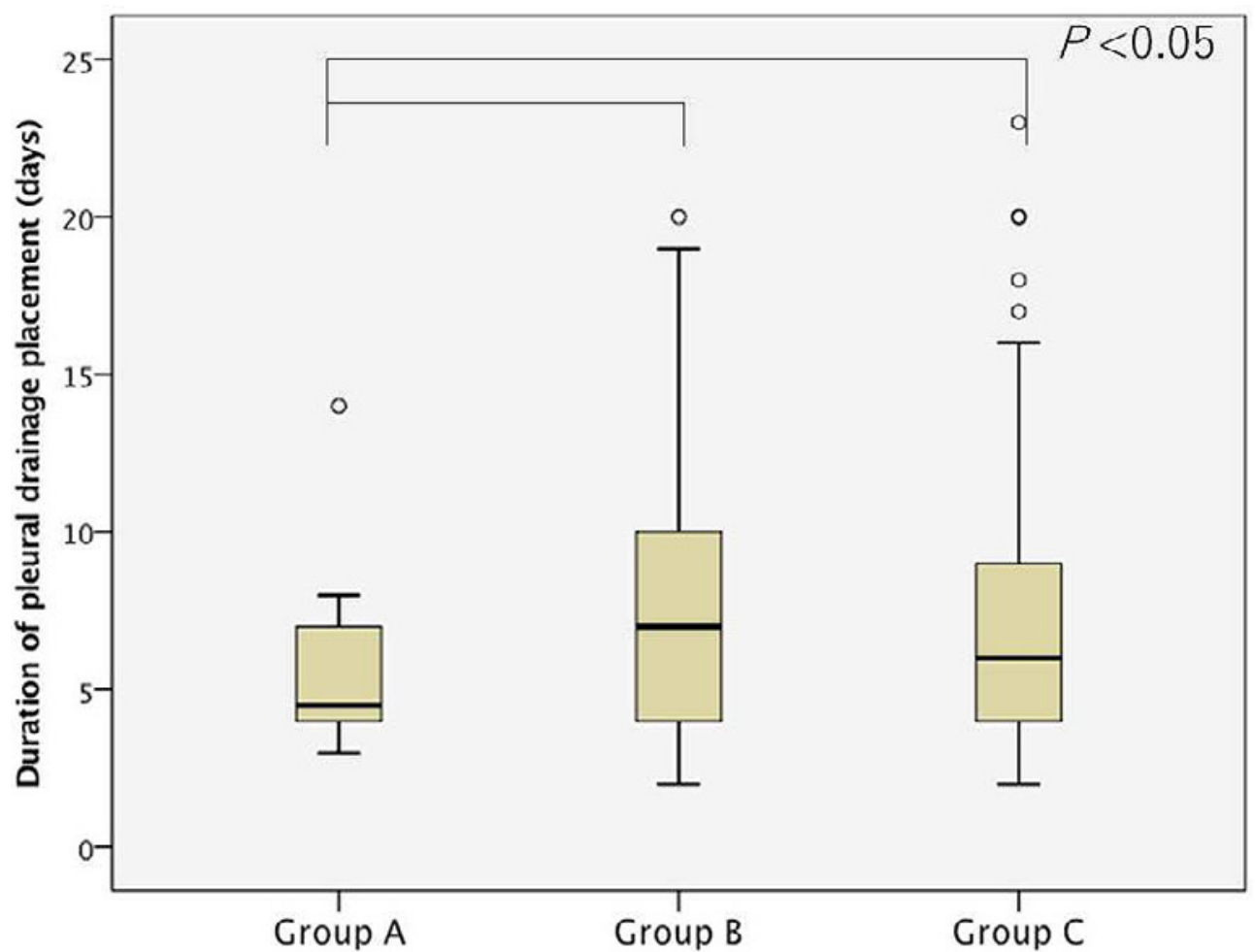

Figure 2. Comparison of the three groups regarding the duration of the pleural drain placement. Group A: APC embolization performed within 30 days before the Fontan. Group B: APC embolization performed more than 30 days before the Fontan. Group C: without APC embolization before the Fontan. The duration of the pleural drain placement in Group A was significantly shorter than that in the other groups $(P<0.05)$.

formula that illustrates fluid translocation between the intravascular and interstitial space, an increase in the hydrostatic pressure or the inability to maintain equilibrium with the oncotic pressure is the primary phenomenon be- hind persistent pleural effusions.

Here, $\mathrm{Cp}$ is defined as $\mathrm{dV} / \mathrm{dP}$, and it clinically depends on the distensibility of the pulmonary vasculature and total blood volume in the pulmonary circulation. ${ }^{13)}$ It 


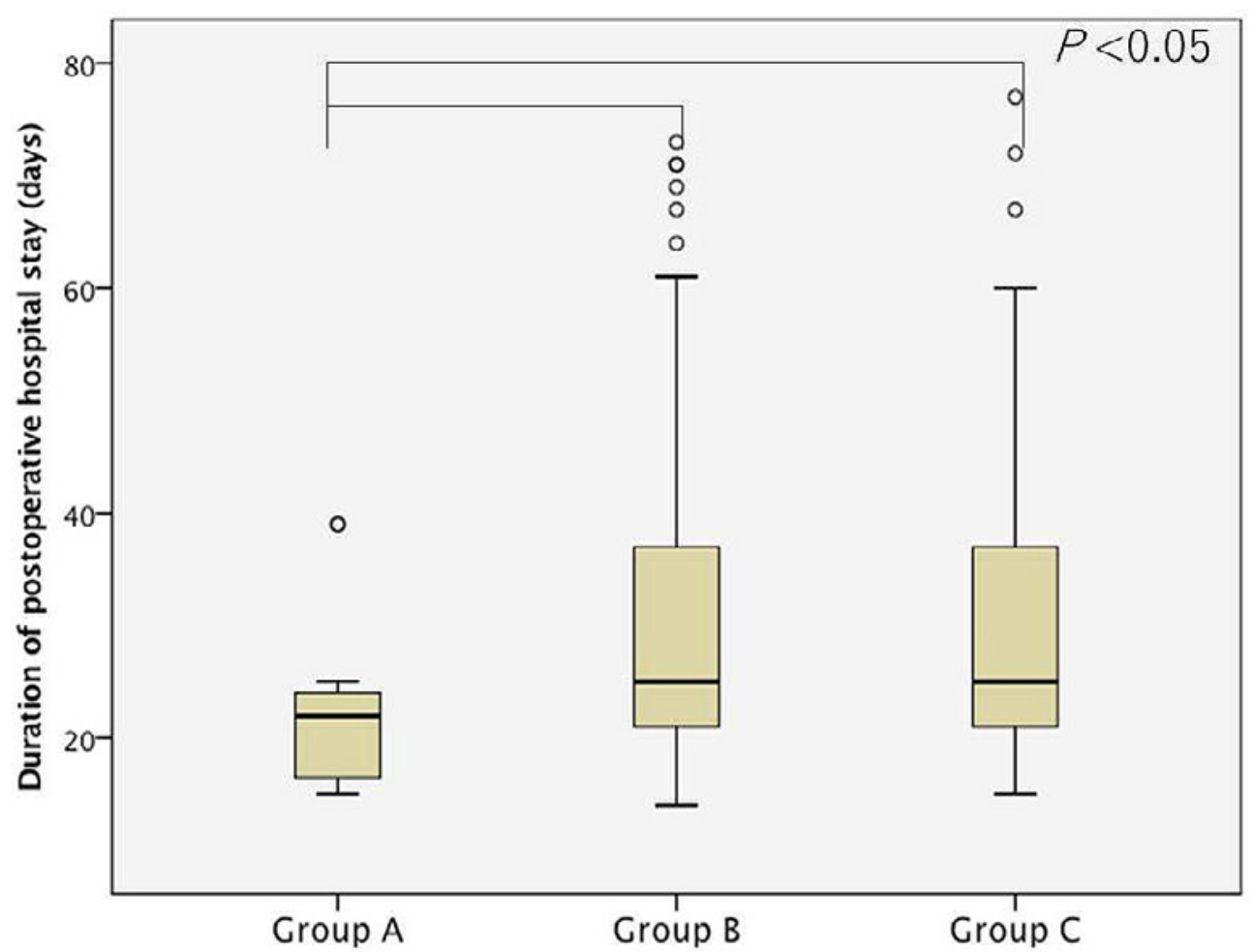

Figure 3. Comparison of the three groups regarding the duration of the postoperative hospital stay. Group A: APC embolization performed within 30 days before the Fontan surgery. Group B: APC embolization performed more than 30 days before the Fontan surgery. Group C: without APC embolization before the Fontan surgery. The duration of the postoperative hospital stay in Group A was significantly shorter than that in the other groups $(P<0.05)$.

might represent the "quality" of pulmonary vascular bed. By contrast, PAI is defined as the sum of the crosssectional area of pulmonary artery branches and might represent the overall "quantity" of the pulmonary vascular bed.

Notably, Senzaki, et al. ${ }^{14)}$ illustrated that there was a significant positive correlation between PAI and $\mathrm{Cp}$. They introduced the equation $\mathrm{Cp}=3 \pi \mathrm{r}^{2} \mathrm{~L}(\mathrm{r} / \mathrm{h}+1)^{2} / \mathrm{E}(2 \mathrm{r} / \mathrm{h}$ +1 ) (r: radius of vessel, L: length of vessel, and $h$ : wall thickness of the vessel) and described that $\mathrm{Cp}$ was determined by the vascular size and distensibility. In that formula, PAI indicated a marker representing the pulmonary vascular size and pulmonary flow. Eyden, et al. ${ }^{13)}$ also described that there was an increase in the $\mathrm{Cp}$ as the flow (and vascular size) increased, probably due to Laplace's law.

Of note, PAI has an advantage of high reliability and a small margin of error because of its simple, clear definition.

The effect of APC coil embolization on adaptation to Fontan circulation: In this study, we demonstrated a possibility that APC coil embolization shortly before Fontan operation exerted a preventive effect against intractable pleural effusions. This effect is completely irrelevant to preoperative PAI because the median value of PAI in Group A was even smaller than those in other groups (Table III).

We deduce that this effect of APC coil embolization is attributed to reduced Qp. According to a report, ${ }^{7)}$ the mean APC flow was $1.59 \pm 0.56 \mathrm{~L} /$ minute $/ \mathrm{m}^{2}$, constituting Qp of $43 \%$. However, beneficial effects of APC coil embolization on the early outcome after a Fontan operation are still under dispute. Banka, et al. ${ }^{15)}$ reported that there was no difference in the hospitalization length between the patients with and without embolizations, but favorable outcomes of APC coil embolization have also been reported. ${ }^{7,16}$

Our data indicated that the favorable effect may be limited to within 30 days before the Fontan operation, probably because the longer the cyanosis is, the greater the new APC that emerges. ${ }^{17)}$ Tolunay, et al. reported that the serum and pulmonary vascular endothelial growth factor (VEGF) levels in cyanotic congenital heart disease have a significant correlation to a poorer pulmonary blood flow. ${ }^{18)}$ According to another in vitro study that illustrated the angiogenic response to VEGF, daughter capillaries and glomeruloid bodies emerged in 1-4 weeks. ${ }^{19)}$ Accordingly, it can be said that APC coil embolization has favorable effects on the adaptation of high-risk cases as long as it is performed shortly before the Fontan operation.

Notably, the influence of these growth factors on the APC development dissolves once the Fontan operation is completed and systemic cyanosis is resolved. A previous report from our institute demonstrated that significant APC either does not emerge or its amount declines after the Fontan operation. ${ }^{10}$ ) From this report, we considered that routine APC coil embolization was not necessary. Considering that APC can resolve after a Fontan opera- 
tion, the main purpose of embolization is to achieve a favorable Fontan circulation. Once an acceptable Fontan circulation (without fenestration) is established, relatively lower CVP can be maintained without embolization thereafter. $^{10)}$

Limitations: A limitation is that this study was a retrospective single-center study. Further, there also were no definite criteria regarding the pleural drainage and hospital discharge in our institute. Regarding the comparison of the patient groups, there were apparently less patients belonging to Group A than those in Group B, possibly because multiple admissions within a short period were often limited by geographical conditions and social insulance programs. Further follow-up is warranted to elucidate the relevance of these factors in correlation with long-term complications and patient survival rates.

\section{Conclusion}

Among the pre-Fontan hemodynamic parameters, PAI was found to be a decisive factor involved in regulating adaptation to a Fontan circulation and that was relevant to the early outcomes after the Fontan operation (e.g., duration of the pleural drainage and length of hospital stay). In the patients with whom pulmonary vascular bed are not abundant, preventive APC coil embolization within 30 days before the Fontan surgery may contribute to the adaptation to Fontan circulation.

\section{Disclosure}

Conflicts of interest: The authors have no financial conflict of interest to disclose.

IRB information: Name of the ethics committee: the Ethics Committee of Fukuoka Children's Hospital. Reference number: 2019-68

\section{References}

1. Nakano T, Kado H, Tatewaki H, et al. Results of extracardiac conduit total cavopulmonary connection in 500 patients. Eur J Cardiothorac Surg 2015; 48: 825-32; discussion 832.

2. Mery CM, Moffett BS, Khan MS, et al. Incidence and treatment of chylothorax after cardiac surgery in children: analysis of a large multi-institution database. J Thorac Cardiovasc Surg 2014 147: 678-86.e1; discussion 685.

3. Gupta A, Daggett C, Behera S, Ferraro M, Wells W, Starnes V. Risk factors for persistent pleural effusions after the extracardiac Fontan procedure. J Thorac Cardiovasc Surg 2004; 127: 1664-9.

4. Ovroutski S, Sohn C, Barikbin P, et al. Analysis of the risk fac- tors for early failure after extracardiac Fontan operation. Ann Thorac Surg 2013; 95: 1409-16.

5. Fu S, Feng ZC, Dietmar S. Factors influencing pleural effusion after Fontan operation: an analysis with 95 patients. Chin Med Sci J 2010; 25: 38-43.

6. Fedderly RT, Whitstone BN, Frisbee SJ, Tweddell JS, Litwin SB. Factors related to pleural effusions after Fontan procedure in the era of fenestration. Circulation 2001; 104(Supplement 1): I148-51.

7. Grosse-Wortmann LG, Drolet C, Dragulescu A, et al. Aortopulmonary collateral flow volume affects early postoperative outcome after Fontan completion: a multimodality study. J Thorac Cardiovasc Surg 2012; 144: 1329-36.

8. Nakata S, Imai Y, Takanashi Y, et al. A new method for the quantitative standardization of cross-sectional areas of the pulmonary arteries in congenital heart diseases with decreased pulmonary blood flow. J Thorac Cardiovasc Surg 1984; 88: 610-9.

9. Ishikawa Y, Ishikawa S, Sagawa K, Ushinohama H, Nakamura M, Kado H. Favorable effects of oxygen inhalation in patients after bidirectional Glenn procedure as assessed by cardiovascular magnetic resonance flow measurement. Circ J 2016; 80: 1378-85.

10. Go K, Kuraoka A, Kodama Y, et al. Transition of aortopulmonary collateral arteries before and after the Fontan procedure. Ped Cardiol Card Surg 2018; 34: 182-8.

11. Yasuhara J, Kuno T, Taki M, et al. Predictors of early postoperative supraventricular tachyarrhythmias in children after the Fontan procedure. Int Heart J 2019; 60: 1358-65.

12. Fukunishi T, Oka N, Yoshii T, et al. Early extubation in the operating room after congenital open-heart surgery. Int Heart $\mathrm{J}$ 2018; 59: 94-8.

13. Vanden Eynden F, Bové T, Chirade M, Van Nooten G, Segers P. Measuring pulmonary arterial compliance: mission impossible? Insights from a novel in vivo continuous-flow based experimental model. Pulm Circ 2018; 8: 1-12.

14. Senzaki H, Kato H, Akagi M, Hishi T, Yanagisawa M. Relationship between the pulmonary artery index and physiological properties of the pulmonary vascular bed. Jpn Circ J 1996; 60: 334-40.

15. Banka P, Sleeper LA, Atz AM, et al. Practice variability and outcomes of coil embolization of aortopulmonary collaterals before Fontan completion: a report from the Pediatric Heart Network Fontan Cross-Sectional Study. Am Heart J 2011; 162: 125-30.

16. Glatz AC, Rome JJ, Small AJ, et al. Systemic-to-pulmonary collateral flow, as measured by cardiac magnetic resonance imaging, is associated with acute post-Fontan clinical outcomes. Circ Cardiovasc Imaging 2012; 5: 218-25.

17. Starnes SL, Duncan BW, Kneebone JM, et al. Vascular endothelial growth factor and basic fibroblast growth factor in children with cyanotic congenital heart disease. J Thorac Cardiovasc Surg 2000; 119: 534-9.

18. Tolunay I, Tunaoglu S, Akyürek N, Halid V, Olgunturk R, Kula S. Serum and pulmonary vascular endothelial growth factor/receptors and haemodynamic measurements in cyanotic congenital heart disease with decreased pulmonary blood flow. Cardiol Young 2011; 21: 608-15.

19. Dvorak HF. Angiogenesis: update 2005. J Thromb Haemost 2005; 3: $1835-42$. 